\title{
GENETICS
}

\section{DRB1*15 allele associated with PR3-ANCA disease}

A genetic analysis has suggested that the major histocompatibility complex (MHC) class II allele $D R B 1^{\star} 15$ is associated with proteinase 3 antineutrophil cytoplasmic autoantibody (PR3-ANCA) disease, particularly in African American people.

During a previous study, the same research team noted that $D R B 1^{\star} 15$ was overrepresented in patients with PR3-ANCA disease. "These results indicated that a study of a larger cohort was required to determine if a statistical association existed between the $D R B 1^{\star} 15$ allele and PR3-ANCA disease," explains corresponding author Gloria Preston. The researchers also found that the $D R B 1^{*} 15$ allele was present in all three of the African American patients with PR3-ANCA disease in their study, a particularly interesting finding as ANCA disease is very rare in African Americans. This finding motivated the researchers to determine whether the $D R B 1^{\star} 15$ allele predisposes African American people to develop ANCA disease.
The present study involved 137 patients with ANCA disease, 41 of whom were African American (16 with PR3-ANCA disease and 25 with myeloperoxidase [MPO]-ANCA disease) and 96 of whom were Caucasian (74 with PR3ANCA disease and 22 with MPO-ANCA disease). The researchers genotyped DNA samples from these patients at the $D R B 1$ and $D Q B 1$ loci using lowresolution analysis. They then used the relative predispositional effects method to analyze the data. "We learned from experienced statisticians that this method is the best way to analyze human leukocyte antigen allele data," states Preston. "It is a well-accepted approach to use when a disease is associated with two or more alleles of a locus."

The $D R B 1^{\star} 15$ allele was identified as being a risk factor for PR3-ANCA: African American individuals with PR3-ANCA were 73 times more likely to have the $D R B 1^{\star} 15$ allele than were community-based controls, and
Caucasian individuals with PR3-ANCA were twice as likely to have the allele. The allele was not found to be associated with MPO-ANCA disease. Preston et al. validated their findings using data from the Vasculitis Clinical Research Consortium: an analysis of combined data from the two cohorts confirmed the finding that the $D R B 1^{\star} 15$ allele is a risk factor for PR3-ANCA disease in African Americans. The researchers also found that a disproportionate number of African American patients carried the $D R B 1^{\star} 1501$ allele of Caucasian descent rather than the $D R B 1^{\star} 1503$ allele of African descent.

The authors hope that their study findings will prompt clinicians to consider the genetic basis of PR3-ANCA in African American patients with this condition.

\section{Claire Greenhill}

Original article $\mathrm{Cao}, \mathrm{Y}$. et al. DRB $1 * 15$ allele is a risk factor for PR3-ANCA disease in African Americans. J. Am. Soc. Nephrol. 22, 1161-1167 (2011) 